\title{
Going Round in Circles with a Multisystemic Disease: A Unique Case of Parasitic Aortitis
}

\author{
Maria Lobo Antunes ${ }^{1,2}$, Gonçalo Cabral ${ }^{3}$, Raquel Tavares ${ }^{4}$, Carla Noronha $^{1,2}$, José Araújo ${ }^{1}$ \\ ${ }^{1}$ Autoimmune Diseases Unit, Hospital Beatriz Ângelo, Loures, Portugal \\ ${ }^{2}$ Internal Medicine Department, Hospital Beatriz Ângelo, Loures, Portugal \\ ${ }^{3}$ Vascular Surgery Department, Hospital Beatriz Ângelo, Loures, Portugal \\ ${ }^{4}$ Infectious Diseases Department, Hospital Beatriz Ângelo, Loures, Portugal
}

Received: $16 / 02 / 2017$

Accepted: 08/05/2017

Published: $17 / 05 / 2017$

How to cite this article: Antunes M, Cabral G, Tavares R, Noronha C, Araújo J. Going round in circles with a multisystemic disease: a unique case of parasitic aortitis. EJCRIM 2017;4: doi:10.12890/2017_000601

Conflicts of Interests: The Authors declare that there are no competing interests.

This article is licensed under a Commons Attribution Non-Commercial 4.0 License

\section{ABSTRACT}

Aortitis results from aortic inflammation, frequent causes being infections and rheumatological disorders. The authors report the case of a 33-year-old black male with recent arterial hypertension, who presented with recurrent abdominal pain, jaundice, anorexia, weight loss and diarrhoea. Laboratory work-up was compatible with inflammatory anaemia and obstructive jaundice, while abdominal imaging revealed a dilated biliary tract, no visible gallstones, cephalic pancreatic globosity and aortic thickening. Pancreatic aspirate was negative for malignant cells, bacteria and Mycobacterium tuberculosis. The jaundice spontaneously subsided and the pancreatic globosity improved over time. Following positive PPD and IGRA, isoniazid was started. However, follow-up investigations revealed a severe bulbar stenosis with intense eosinophilic infiltrate, multiple non-necrotizing granulomas, and thoracic and abdominal aortitis not previously recognized. Immunological profile (ECA, ANCA and IgG4), eggs and parasites in stool samples were negative. The multisystemic disease, with an insidious and migrating behaviour, gastrointestinal and vascular involvement, granulomatous inflammatory response and tissue eosinophilia, raised the suspicion of a parasitic infestation (despite negative screening) or vasculitis. After 7 days of empirical treatment with albendazole and ivermectin, the patient passed a specimen of Ascaris lumbricoides in the stool and improved clinically.

\section{LEARNING POINTS}

- Parasitic infestations can cause aortitis: negative stool screening does not rule out roundworm infestation and granulomatous inflammatory response with eosinophilia is a histological hallmark.

- Infestation by Ascaris lumbricoides can have multisystemic expression and mimic rheumatological disorders such as vasculitis.

- This is the first report of a parasitic-related aortitis in humans.

\section{KEYWORDS}

Aortitis, Ascaris lumbricoides, eosinophilic inflammation 


\section{CASE DESCRIPTION}

A 33-year-old black male, originally from São Tomé and Príncipe, presented to the emergency department because of recurrent abdominal pain in his left upper quadrant which had started 6 months previously, jaundice for the past 3 weeks, anorexia, weight loss (4 kg in 6 months) and diarrhoea with occasional haematochezia. He denied other symptoms including fever, dyspnoea, cough and sputum.

The patient had a history of arterial hypertension, recently diagnosed, with an apparently normal secondary hypertension work-up. He denied alcohol intake, smoking, drug abuse, allergies, recent travelling and close contact with animals.

Clinically there was evident jaundice, no fever, and normal vital signs and cardio-pulmonary and abdominal examinations.

Laboratory data revealed mild microcytic anaemia (11.3 g/dl), an elevated erythrocyte sedimentation rate (61 mm/1st hour) and C-reactive protein $(4.4 \mathrm{mg} / \mathrm{dl})$, a total bilirubin of $6.25 \mathrm{mg} / \mathrm{dl}$ with a conjugated bilirubin of $5.65 \mathrm{mg} / \mathrm{dl}$, and mildly elevated liver enzymes. Coagulation, renal function, amylase and chest X-ray were normal.

An abdominal CT scan showed a dilated biliary tract, with no visible gallstones, pancreatic globosity with enlarged abdominal lymph nodes (hepatic hilum, celiac trunk and adjacent to the pancreatic head), hepatomegaly with regular borders, splenomegaly, and discrete aortic thickening. Abdominal MRI confirmed intra-hepatic and common bile duct ectasia $(\sim 10 \mathrm{~mm})$ together with marked globosity of the pancreatic head and proximal portion of the body (Fig. 1).

Endoscopic ultrasound with cytological and bacteriological examination of the pancreatic head and lymph nodes were negative for malignant cells, bacteria and Mycobacterium tuberculosis. A colonoscopy was performed but was inconclusive (inadequate intestinal preparation), with normal biopsies.

The immunological profile was negative for ANA, dsDNA, ENA, AMA, ASMA, anti-LKM, ECA and ANCA. IgG4 was 91.2 mg/dl (reference range 3.0-201 mg/dl). HIV, HCV, HBV, HSV, CMV and EBV serologies were also negative. Tumour marker CA 19-9 was not elevated. Stool samples for microbiological culture, cysts, eggs and parasite examination, and blood cultures were all negative as well.

Curiously, the jaundice subsided spontaneously in 2 days and the abdominal pain improved.

Positive PPD and IGRA tests suggested latent tuberculosis so the patient was started on isoniazid.

A follow-up endoscopic ultrasound 4 months later revealed severe bulbar stenosis which had not been previously present and was clinically asymptomatic. Duodenal biopsy disclosed an intense eosinophilic inflammatory infiltrate, and non-necrotizing granulomas with negative Ziehl-Neelsen staining (Fig. 2).

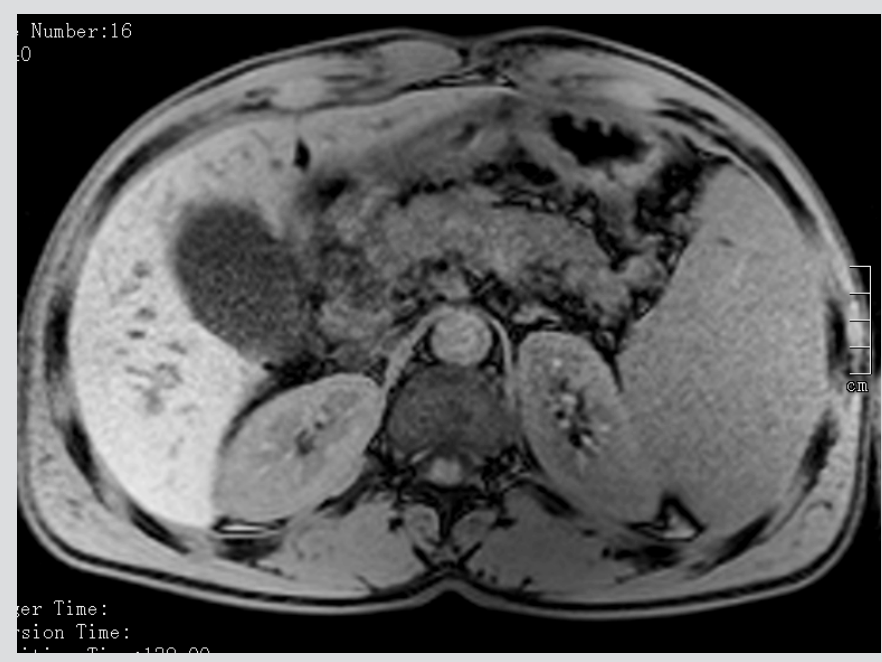

Figure 1. Abdominal MRI with marked globosity of the pancreatic head and proximal portion of the body

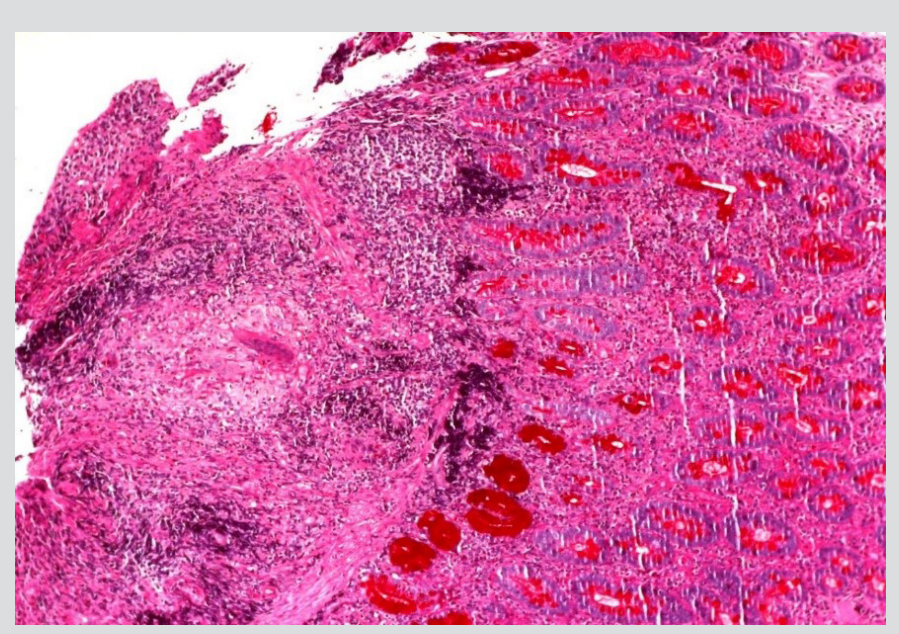

Figure 2. Bulbar biopsy showing non-necrotizing granulomata with an intense eosinophilic inflammatory infiltrate

A contrast enhanced abdominal CT scan disclosed 50\% stenosis of the left renal artery, and lower thoracic and abdominal aorta thickening, compatible with aortitis (Fig. 3). Abdominal lymph nodes and the hepatosplenomegaly remained unchanged, but consistent improvement of the pancreatic globosity was observed. 
A multidisciplinary discussion suggested an insidious and migrating eosinophilic granulomatous disease with pancreatic, biliary tract, duodenum and vascular involvement. The differential diagnosis included parasitic infestation, large-vessel vasculitis, sarcoidosis and lymphoproliferative disease. Exploratory laparoscopy was deferred. It was decided to repeat serological and stool sample screening and to start empirical treatment with albendazole and ivermectin for 7 days; if negative, corticosteroids were to be started, based on the presumptive diagnosis of an autoimmune disorder. However, on the 7th day of treatment, the patient spontaneously passed a parasite in stool (Fig. 4). A serological panel for parasites was positive for Ascaris lumbricoides.

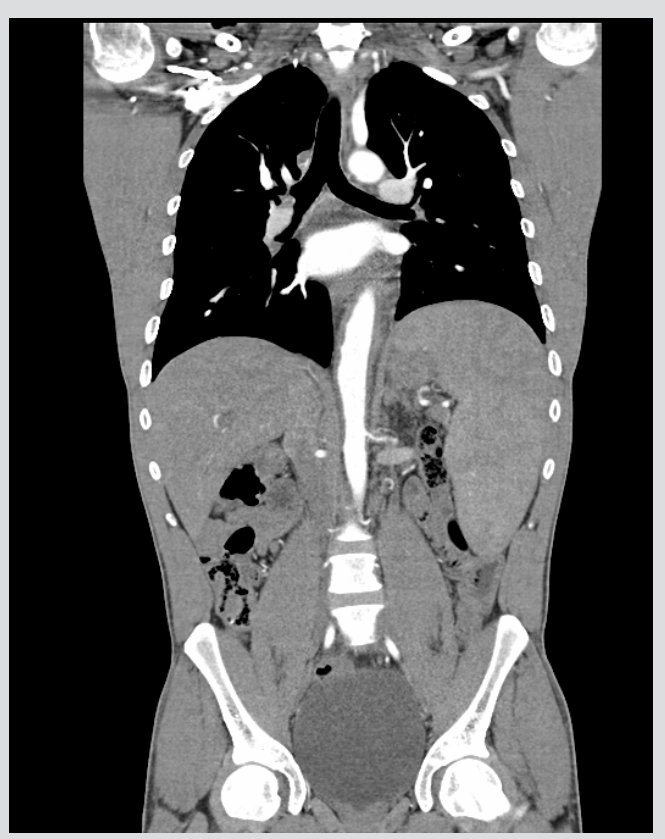

Figure 3. Contrast enhanced CT abdominal scan showing left renal artery stenosis, aortitis and hepatosplenomegaly

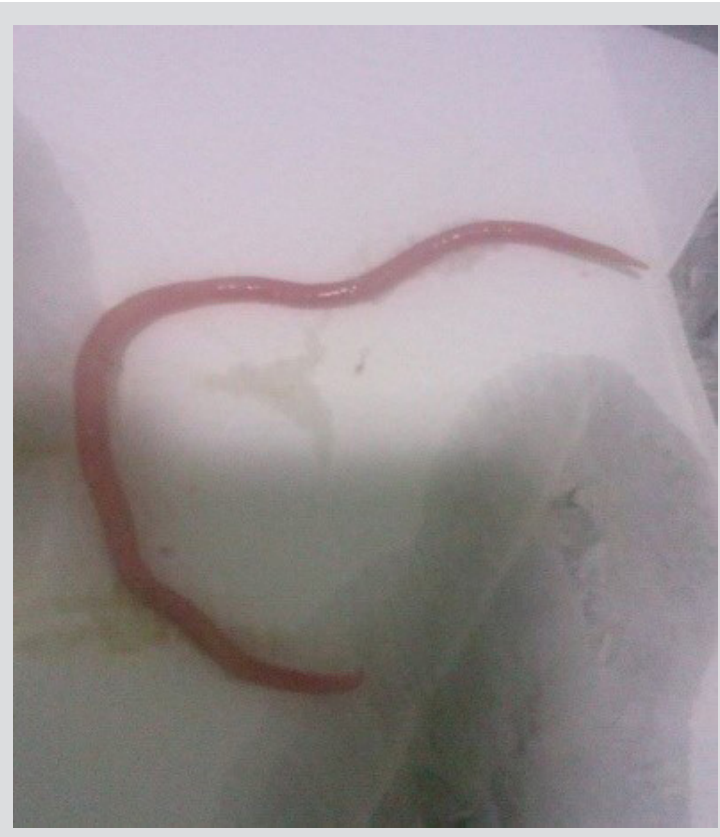

Figure 4. Adult Ascaris lumbricoides specimen passed in the stool of the patient

In conclusion, $A$. lumbricoides infestation with multisystemic involvement, including aortitis, was diagnosed.

The renal artery stenosis was corrected by stenting and allowed discontinuation of anti-hypertensive medication.

\section{DISCUSSION}

Aortitis is the pathological term for inflammation of the aortic wall[1]. The classification of aortitis broadly includes underlying rheumatological and infectious diseases, along with isolated aortitis ${ }^{[1]}$. Aortitis is a possible presentation of vasculitis, an inflammatory aortic condition that cannot be attributed to atherosclerosis alone ${ }^{[1,2]}$. The 2012 Revised Chapel Hill Consensus Conference Nomenclature of Vasculitides recognizes two major non-infectious aortitis variants: Takayasu's arteritis and giant cell arteritis ${ }^{[2,3]}$. A number of organisms have been associated with infectious aortitis, including bacteria (Staphylococcus, Salmonella, Streptococcus pneumoniae, Treponema pallidum), mycobacteria ${ }^{[4]}$ and viruses (hepatitis B and C, Epstein-Barr, cytomegalovirus) ${ }^{[5]}$. Multiple different parasitic infections have also occasionally been associated with rheumatic syndromes, such as reactive arthritis, spondyloarthropathy and vasculitis ${ }^{[6]}$. Parasitic infestations are frequent, but vasculitis related to parasitosis is very rare ${ }^{[7]}$. This may be the consequence of direct parasite infiltration of the vascular wall, or secondary to immune response $\mathrm{e}^{[7]}$.

Aortitis associated with parasitic infection has rarely been reported in the literature, with a few cases in cattle in Iraq ${ }^{[8]}$, and never associated with A. lumbricoides. A thorough PubMed search revealed no case report of parasitic-related aortitis in humans.

A. lumbricoides is the most frequent cause of parasitosis worldwide ${ }^{[6,9]}$. Adult worms may live in the gut for up to 6-18 months, causing bowel obstruction ${ }^{[10]}$ and migrating into the appendix, hepatobiliary system or pancreatic ducts with attending appendicitis, biliary colic, cholangitis, cholecystitis, hepatic abscesses and acute pancreatitis ${ }^{[11]}$. The histological hallmark of parasitic diseases is mainly a granulomatous inflammation ${ }^{[12]}$. Most cases of ascariasis are asymptomatic with symptoms largely restricted to individuals with a large worm load ${ }^{[13]}$. The diagnosis of ascariasis is usually made by stool microscopy, while eggs may be seen on direct examination of faeces or following concentration techniques ${ }^{[14]}$. Early diagnosis can be difficult and negative screening does not rule out infestation. 
Laboratory findings include peripheral eosinophilia, while serum levels of IgG and IgE can be elevated during early infection ${ }^{[15]}$.

Obstructive jaundice with a dilated biliary tract, but no gallbladder stones, in this patient, was probably due to worm invasion, and quickly and spontaneously subsided after parasite migration into the intestine.

The insidious, fluctuating and migratory behaviour of the disease in this case, affecting the upper gastrointestinal tract, biliary tract and pancreas and the eosinophilic granulomatous inflammatory response, raised suspicion of a parasite infestation, although aortitis is a very uncommon presentation. We can only speculate that aortitis and renal artery stenosis could have been caused either by direct parasite invasion through a possible perforated bowel, not revealed by examination, or by a secondary immunoreactive response. In a global world, with more people travelling, the prevalence of ascariasis will probably increase in developed countries.

\section{REFERENCES}

1. Gornik HL, Creager MA. Aortitis. Circulation 2008;117:3039-3051.

2. Ladich E, Yahagi K, Romero ME, Virmani R. Vascular diseases: aortitis, aortic aneurysms, and vascular calcification. Cardiovasc Pathol 2016;25:432-441.

3. Jennette JC, Falk RJ, Bacon PA, Basu N, Cid MC, Ferrario F, et al. 2012 Revised international Chapel Hill consensus conference nomenclature of vasculitides. Arthritis Rheum 2013;65:1-11.

4. Töpel I, Zorger N, Steinbauer M. Inflammatory diseases of the aorta Part 2: infectious aortitis. Gefässchirurgie 2016;21(Suppl 2):S87-S93.

5. Grajales C, Pineda JC. Pathophysiological relationship between infections and systemic vasculitis. Autoimmune Dis 2015;2015:286783.

6. Lhote F. Systemic vasculitis during parasitosis. Presse Med 2004;33(19 Pt2):1389-13401.

7. Peng SL. Rheumatic manifestations of parasitic diseases. Semin Arthritis Rheum 2002:31:228-247.

8. Al-Zubaidy AJ. Observations on parasitic aortitis in cattle in Iraq. Trans R Soc Trop Med Hyg 1973;67:436.

9. Diouf C, Kane A, Ndoye NA, Ndour O, Faye-Fall AL, Fall M, et al. Volvulus of the small bowel due to Ascaris lumbricoides package: about a case. Pan Afr Med J 2016;24:208.

10. Khuroo MS, Rather AA, Khuroo NS, Khuroo MS. Hepatobiliary and pancreatic ascariasis. World J Gastroenterol 2016;22:7507-7517.

11. Sundriyal D, Mittal G, Kumar S, Manjunath S, Sharma N, Gupta M. Biliary ascariasis mimicking colonic tumor infiltration of the biliary system. Indian J Surg Oncol 2015;6:280281.

12. Papparella S. Histology in diagnosis of parasitic diseases. Parasitologia 2004;46:157-158.

13. Khuroo MS. Ascariasis. Gastroenterol Clin North Am 1996;25:553-577.

14. Tietze, PE, Tietze, PH. The roundworm, Ascaris lumbricoides. Prim Care 1991;18:25.

15. Weller, PF. Eosinophilia in travelers. Med Clin North Am 1992;76:1413. 